\title{
Fluorescent Assay Infectious Dose Unit
}

National Cancer Institute

\section{Source}

National Cancer Institute. Fluorescent Assay Infectious Dose Unit. NCI Thesaurus. Code C68880.

A unit for measurement of infectious dose as defined in the particular fluorescent assay method. 